\title{
Органічна хімія
}

УДК 547.732.7+ 547.793.4

\section{SYNTHESIS OF NOVEL 3-(1,2,4-OXADIAZOL-5-YL)THIOPHEN-2-AMINE DERIVATIVES VIA GEWALD REACTION}

\section{O. Shyyka*, M. Svyrydenko, M. Tupychak, N. Pokhodylo, M. Obushak}

Ivan Franko National University of Lviv, Kyryla i Mefodiya Str., 6, 79005 Lviv, Ukraine e-mail: shiyka.olya@gmail.com

Novel substituted 3-(1,2,4-oxadiazol-5-yl)thiophen-2-amines were obtained by the multicomponent Gewald reaction of an activated nitrile with 1,2,4-oxadiazole moiety, a carbonyl component and elemental sulphur in the presence of morpholine as a catalyst.

Keywords: 2-aminothiophenes, 1,2,4-oxadiazole, Gewald reaction, multicomponent reaction.

DOI: https:/doi.org/10.30970/vch.6201.183

\section{Introduction}

Gewald multicomponent reaction has received much attention because of its elegance, versatility, convenience, and effectiveness [1,2]. A number of methods for the synthesis of 2-aminothiophene derivatives has been developed using such strategy and a wide range of functionalized 2-aminothiophenes has been prepared [1-4]. Moreover, produced by Gewald reaction 2-aminothiophenes are important five-membered heterocyclic building blocks in organic synthesis, and the chemistry of these small molecules is still developing [3]. Thiophene derivatives are utilized in industrial chemistry and material science as corrosion inhibitors, organic semiconductors, organic field-effect transistors (OFETs) [5], organic light-emitting diodes (OLEDs) [6], etc. Still special attention is given to biologically and pharmacologically attractive aminothiophene derivatives. Till now, 2aminothiophene scaffold prove to act as efficient synthon for the synthesis of biological active thiophene-containing heterocycles $[3,7]$.

From the other point of view, 1,3,4-oxadiazoles and 1,2,4-oxadiazoles derivatives are widely investigated and represent another important scaffold for current drug discovery due to the remarkable biological properties possessed by oxadiazole derivatives $[8,9]$. The oxadiazole scaffold is found in a series of compounds with anticonvulsant, antiinflammatory, antiallergic, antipsychotic, antimicrobial, antitumor, antiviral and antituberculosis activity [10]. Some of them also act as antidepressants and analgesics. Therefore, both the development of the synthesis of new oxadiazole derivatives and evaluation of their biological activity are in high demand.

Our previous studies on aminothiophenes established some convenient chemical modifications of the Gewald 2-aminothiophenes and isomeric 3-aminothiophenes, the scope of their synthetic application in various fused nitrogen containing heterocycles preparation

(C) Shyyka O., Svyrydenko M., Tupychak M. et al., 2021 
in energy-saving and environmentally friendly manner was also determined [11-19]. Such approaches allowed to create broad combinatorial libraries for drug discovery and to select drug-like examples possessing significant anticancer activity [20-22].

Thus, synthesis of novel functionalized 3-(1,2,4-oxadiazol-5-yl)thiophen-2-amines will broaden the combinatorial library with the aim of the anticancer agent search.

\section{Results and discussion}

Herein, based on multicomponent Gewald method [23] a number of new 3-(5-aryl1,3,4-oxadiazol-2-yl)thiophen-2-amines 3a-j were obtained with high yield. Proposed protocol involves multicomponent condensation of an activated nitrile, a carbonyl component and elemental sulphur in the presence of morpholine as a catalyst. The facility of one-pot multicomponent Gewald rection also allows to implement the variety of carbonyl components as well as nitriles with substituents of different nature.<smiles>[R]CC([R])=O</smiles>

1a,b<smiles>[R]c1noc(CC#N)n1</smiles>

2a-e<smiles>[R]c1noc(-c2c(N)sc([R])c2[R])n1</smiles>

3a-j

1: $\mathrm{R}^{1}=\mathrm{R}^{2}=\mathrm{Me}(\mathbf{b}), \mathrm{R}^{1}+\mathrm{R}^{2}=-\left(\mathrm{CH}_{2}\right)_{4^{-}}(\mathbf{a})$

2: $\mathrm{R}^{3}=\mathrm{Ph}(\mathbf{a}), 2-\mathrm{MeC}_{6} \mathrm{H}_{4}(\mathbf{b}), 4-\mathrm{FC}_{6} \mathrm{H}_{4}(\mathbf{c}), 2-\mathrm{ClC}_{6} \mathrm{H}_{4}(\mathbf{d}), 4-\mathrm{ClC}_{6} \mathrm{H}_{4}(\mathbf{e})$

Gewald reaction of carbonyl compoumds 1a, $\mathbf{b}$ with (1,2,4-oxadiazol-5-yl)acetonitriles 2a-e Реакція Гевальда карбонільних сполук 1a, b 3 (1,2,4-оксадіазол-5-іл)ацетонітрилами 2a-e

To determine the possibility of successful implementation of new heterocyclic

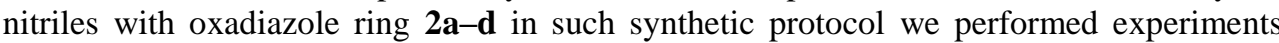
with well-established model carbonyl components such as ethyl 3-oxobutanoate 1a and cyclohexanone $\mathbf{1}$. On contrary, mentioned starting (1,2,4-oxadiazol-5-yl) acetonitriles 2a-e (Table 1) were studied in Gewald protocol for the first time to determine if 1,2,4-oxadiazole ring is a good acceptor suitable for Knoevenagel condensation and does the proposed conditions tolerates 1,2,4-oxadiazol moiety at all. (1,2,4-Oxadiazol-5-yl)acetonitriles with both donor and acceptor substituents were recently described by our group and the scope of their application in green click protocols with azides was established [24]. The experiment showed that these conversations were performed in good yields.

Table 1

Nitriles 2a-e

Нітрили 2a-e

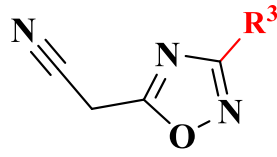

\begin{tabular}{|c|c|c|c|c|c|}
\hline ID & 2a & 2b & 2c & 2d & 2e \\
\hline$R^{3}$ & &
\end{tabular}


We found out that reaction proceeds smoothly and desired functionalized aminothiophenes with additional heterocyclic ring -1,2,4-oxadiazole 3a-j were obtained as crystalline compounds of high purity and with good yields (Table 2). The structure of isolated products was confirmed by ${ }^{1} \mathrm{H}$ and ${ }^{13} \mathrm{C}$ NMR spectroscopy. Utilization of $(1,2,4-$ oxadiazol-5-yl)acetonitriles and ketones allowed to form a series of previously undescribed aminothiophenes, suitable for biological screening or further modification.

It should be mentioned, that the utility of easily accessible nitriles with 1,2,4oxadiazole allows to vary the substituent in aryl core, what would broaden combinatorial library of biologically suitable 1,2,4-oxadiazolyl-thiophene derivatives.

Moreover, the synthesized 3-(aryl)-1,2,4-oxadiazol-5-yl)-thiophen-2-amines 3a-j were submitted and selected by NCI (National Cancer Institute, USA) for evaluation at the single concentration towards a panel of the approximately sixty cancer cell lines among nine different cancer types: leukemia, non-small cell lung, colon, CNS, melanoma, ovarian, renal, prostate and breast cancers (http://dtp.nci.nih.gov).

The obtained compounds were also selected by Community for Open Antimicrobial Drug Discovery (CO-ADD) (UK, Australia) to test antimicrobial properties. The results of biological experiments will be reported in a due course.

\section{Conclusion}

In the current work, we investigated the one-pot multicomponent Gewald protocol, which made possible to easily synthesize a range of functionalized 1,2,4-oxadiazolyl aminothiophene compounds suitable for screening on biological activity in cost and time effective manner from available reagents and ability to vary substituents. The products - 3(aryl)-1,2,4-oxadiazol-5-yl)-thiophen-2-amines were obtained for the first time in good yields and high purity. Moreover, present amino group in obtained Gewald thiophenes allows further modification on demand to improve pharmacologic profile of substances.

\section{Experimental part}

${ }^{1} \mathrm{H}$ and ${ }^{13} \mathrm{C}$ NMR spectra were recorded on Varian Unity Plus 400 (400 and $101 \mathrm{MHz}$, respectively), Bruker 170 Avance $500(500$ and $126 \mathrm{MHz}$, respectively) spectrometers in DMSO- $d_{6}$ solutions using TMS or the deuterated solvent as internal reference. Elemental analyses were accomplished using a Carlo Erba 1106 instrument. Melting points were determined on a Boetius melting point apparatus.

\section{General Procedure for the synthesis of 2-aminothiophenes 3a-j}

Elemental sulphur $(0,01 \mathrm{~mol})$ and morpholine $(0,8 \mathrm{ml})$ was added to a solution of carbonyl component 1a,b $(0,01 \mathrm{~mol})$ and activated with oxadiazole ring nitrile 2a-e $(0,01 \mathrm{~mol})$ in $\mathrm{EtOH}$. The mixture was stirred and heated to $50-60^{\circ} \mathrm{C}$ at reflux for $3 \mathrm{~h}$. The reaction mixture was cooled to the room temperature and the formed solid was filtered off and recrystallized from ethanol.

4,5-Dimethyl-3-(3-phenyl-1,2,4-oxadiazol-5-yl)thiophen-2-amine 3a. Yield $81 \%$. M.p. $=164-165{ }^{\circ} \mathrm{C} .{ }^{1} \mathrm{H}$ NMR (400 MHz, DMSO- $\left.d_{6}\right)$ ppm: $\delta 2,15$ (s, 3H, Me), 2,25 (s, 3H, $\mathrm{Me}$ ), 7,53-7,75 (m, 3H, $\left.\mathrm{H}_{\mathrm{Ph}}-3,4,5\right), 7,80$ (br. s, $2 \mathrm{H}, \mathrm{NH}_{2}$ ), 8,10-8,16 (m, 2H, $\left.\mathrm{H}_{\mathrm{Ph}}-2,6\right) \cdot{ }^{13} \mathrm{C}$ NMR (101 MHz, DMSO-d $\left.d_{6}\right)$ ppm: $\delta 173,40\left(\mathrm{C}_{\text {Oxadz }}-5\right), 166,58\left(\mathrm{C}_{\text {Oxadz }}-3\right), 160,31\left(\mathrm{C}_{\mathrm{Th}}\right)$, $131,70\left(\mathrm{C}_{\mathrm{Th}}\right), 129,51\left(2 \mathrm{xC}_{\mathrm{Ph}}-3,5\right), 128,04\left(\mathrm{C}_{\mathrm{Ph}}-4\right), 127,68\left(2 \mathrm{xC}_{\mathrm{Ph}}-2,6\right), 127,13\left(\mathrm{C}_{\mathrm{Ph}}-1\right)$, 114,52 $\left(\mathrm{C}_{\mathrm{Th}}\right), 99,02\left(\mathrm{C}_{\mathrm{Th}}\right), 14,42(\mathrm{Me}), 12,44(\mathrm{Me})$. Found, \%: C 61,94; $\mathrm{H} \mathrm{4,85;} \mathrm{N}$ 15,53. $\mathrm{C}_{14} \mathrm{H}_{13} \mathrm{~N}_{3} \mathrm{OS}$. Calculated, C 61,97; H 4,83; N 15,49. 
O. Shyyka, M. Svyrydenko, M. Tupychak et al ISSN 2078-5615. Visnyk of the Lviv University. Series Chemistry. 2021. Issue 62

Yields and melting point of synthesized compounds $\mathbf{3 a - j}$

Table 2

Таблиия 2

Виходи і температури плавлення синтезованих сполук 3a-j

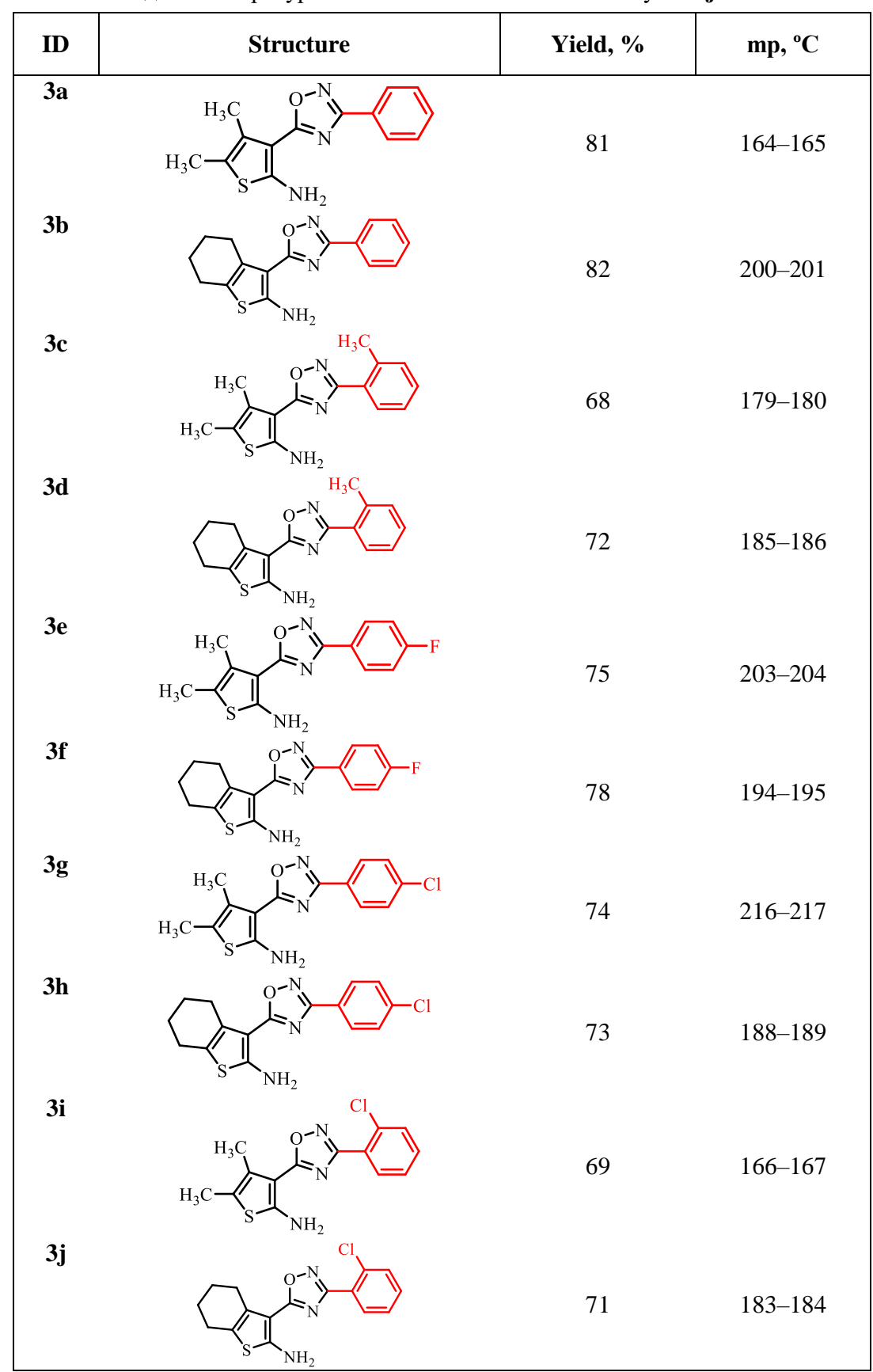


O. Shyyka, M. Svyrydenko, M. Tupychak et al.

3-(3-Phenyl-1,2,4-oxadiazol-5-yl)-4,5,6,7-tetrahydrobenzo[b]thiophen-2-amine 3b. Yield $82 \%$. M.p. $=200-201{ }^{\circ} \mathrm{C} .{ }^{1} \mathrm{HNMR}\left(400 \mathrm{MHz}, \mathrm{DMSO}-d_{6}\right)$ ppm: $\delta 1,73$ (br. s, 4H, $\mathrm{CH}_{2}$ ), 2,45 (br. s, $2 \mathrm{H}, \mathrm{CH}_{2}$ ), 2,73 (br. s, $2 \mathrm{H}, \mathrm{CH}_{2}$ ), 7,51-7,55 (m, 3H, $\mathrm{H}_{\mathrm{Ph}}-3,4,5$ ), 7,68 (br. s, $\left.2 \mathrm{H}, \mathrm{NH}_{2}\right), 8,09-8,11\left(\mathrm{~m}, 2 \mathrm{H}, \mathrm{H}_{\mathrm{Ph}}-2,6\right) .{ }^{13} \mathrm{C}$ NMR (101 MHz, DMSO-d $\left.{ }_{6}\right)$ ppm: $\delta 173,31$ $\left(\mathrm{C}_{\text {Oxadz }}-5\right), 166,58\left(\mathrm{C}_{\text {Oxadz }}-3\right), 160,86\left(\mathrm{C}_{\mathrm{Th}}\right), 131,60\left(\mathrm{C}_{\mathrm{Th}}\right), 130,40\left(\mathrm{C}_{\mathrm{Ph}}-4\right), 129,43\left(2 \mathrm{xC}_{\mathrm{Ph}}-\right.$ 3,5), 127,64 (2xC $\left.\mathrm{Ph}_{\mathrm{Ph}}-2,6\right), 127,17\left(\mathrm{C}_{\mathrm{Ph}}-1\right), 117,87\left(\mathrm{C}_{\mathrm{Th}}\right), 97,74\left(\mathrm{C}_{\mathrm{Th}}\right), 26,17\left(\mathrm{CH}_{2}\right), 24,29$ $\left(\mathrm{CH}_{2}\right), 23,20\left(\mathrm{CH}_{2}\right), 22,67\left(\mathrm{CH}_{2}\right)$. Found, \%: C 64,57; $\mathrm{H} 5,10 ; \mathrm{N} 14,16 . \mathrm{C}_{16} \mathrm{H}_{15} \mathrm{~N}_{3} \mathrm{OS}$. Calculated, C 64,62; H 5,08; N 14,13.

4,5-Dimethyl-3-(3-(o-tolyl)-1,2,4-oxadiazol-5-yl)thiophen-2-amine 3c. Yield 68 \%. M.p. $=179-180{ }^{\circ} \mathrm{C} .{ }^{1} \mathrm{HNMR}\left(400 \mathrm{MHz}, \mathrm{DMSO}-d_{6}\right) \mathrm{ppm}: \delta 2,14\left(\mathrm{~s}, 3 \mathrm{H}, \mathrm{CH}_{3}\right), 2,25$ (s,3H, $\left.\mathrm{CH}_{3}\right), 2,57$ (s,3H, $\left.\mathrm{CH}_{3}\right), 7,33-7,37$ (m, 2H, $\left.\mathrm{H}_{\mathrm{Ar}}-3,5\right), 7,41-7,44\left(\mathrm{~m}, 1 \mathrm{H}, \mathrm{H}_{\mathrm{Ar}}-4\right), 7,59$ (br. s, $\left.2 \mathrm{H}, \mathrm{NH}_{2}\right), 8,03$ (d, $\left.1 \mathrm{H}, J=7,6 \mathrm{~Hz}, \mathrm{H}_{\mathrm{Ar}}-6\right) .{ }^{13} \mathrm{C}$ NMR $\left(101 \mathrm{MHz}, \mathrm{DMSO}-d_{6}\right) \mathrm{ppm}: \delta$ $172,42\left(\mathrm{C}_{\mathrm{Oxadz}}-5\right), 167,21\left(\mathrm{C}_{\mathrm{Oxadz}}-3\right), 160,07\left(\mathrm{C}_{\mathrm{Th}}\right), 137,90\left(\mathrm{C}_{\mathrm{Th}}\right), 131,76\left(\mathrm{C}_{\mathrm{Ar}}\right), 130,96\left(\mathrm{C}_{\mathrm{Ar}}\right)$, $130,34\left(\mathrm{C}_{\mathrm{Ar}}\right), 128,11\left(\mathrm{C}_{\mathrm{Ar}}\right), 126,57\left(\mathrm{C}_{\mathrm{Ar}}\right), 126,46\left(\mathrm{C}_{\mathrm{Ar}}\right), 114,55\left(\mathrm{C}_{\mathrm{Th}}\right), 99,11\left(\mathrm{C}_{\mathrm{Th}}\right), 24,27$ $\left(\mathrm{CH}_{3}\right), 14,39\left(\mathrm{CH}_{3}\right), 12,41\left(\mathrm{CH}_{3}\right)$. Found, \%: C 63,17; H 5,28; N 14,76. $\mathrm{C}_{15} \mathrm{H}_{15} \mathrm{~N}_{3} \mathrm{OS}$. Calculated, C 63,13; H 5,30; N 14,73.

3-(3-(o-Tolyl)-1,2,4-oxadiazol-5-yl)-4,5,6,7-tetrahydrobenzo[b]thiophen-2-amine 3d. Yield $72 \%$. M.p. $=185-186{ }^{\circ} \mathrm{C} .{ }^{1} \mathrm{HNMR}\left(400 \mathrm{MHz}, \mathrm{DMSO}-d_{6}\right)$ ppm: $\delta 1,72$ (br. s, $4 \mathrm{H}$, $\mathrm{CH}_{2}$ ), 2,46 (br. s, $2 \mathrm{H}, \mathrm{CH}_{2}$ ), 2,56 (s,3H, $\left.\mathrm{CH}_{3}\right), 2,71$ (br. s, $\left.2 \mathrm{H}, \mathrm{CH}_{2}\right), 7,34-7,37$ (m, $2 \mathrm{H}, \mathrm{H}_{\mathrm{Ar}}$ 3,5), 7,40-7,43 (m, 1H, $\left.\mathrm{H}_{\mathrm{Ar}^{-}}-4\right)$, 7,57 (br. s, 2H, $\mathrm{NH}_{2}$ ), 8,03 (d, 1H, J = 7,6 Hz, H $\mathrm{Ar}_{\mathrm{Ar}}-6$ ). Found, \%:C 65,61; H 5,48; N 13,51. $\mathrm{C}_{17} \mathrm{H}_{17} \mathrm{~N}_{3} \mathrm{OS}$. Calculated, C 65,57; H 5,50; N 13,49.

3-(3-(4-Fluorophenyl)-1,2,4-oxadiazol-5-yl)-4,5-dimethylthiophen-2-amine $3 \mathrm{e}$. Yield $75 \%$. M.p. $=203-204{ }^{\circ} \mathrm{C} .{ }^{1} \mathrm{HNMR}\left(500 \mathrm{MHz}, \mathrm{DMSO}-d_{6}\right)$ ppm: $\delta 2,13\left(\mathrm{~s}, 3 \mathrm{H}, \mathrm{CH}_{3}\right)$, 2,23 (s,3H, $\mathrm{CH}_{3}$ ), 7,38-7,33 (m, 2H, $\left.\mathrm{H}_{\mathrm{Ar}}-3,5\right), 7,66$ (br. s, $2 \mathrm{H}, \mathrm{NH}_{2}$ ), 8,16-8,24 (m, 2H, $\mathrm{H}_{\mathrm{Ar}}$ 2,6). ${ }^{13} \mathrm{C}$ NMR (126 MHz, DMSO- $\left.d_{6}\right)$ ppm: $\delta$ 173,42 (C $\left.\mathrm{C}_{\text {Oxadz }}-5\right), 165,76\left(\mathrm{C}_{\text {Oxadz }}-3\right), 163,04$ $\left(\mathrm{C}_{\mathrm{Th}}\right), 160,40\left(\mathrm{~d},{ }^{1} J_{C-F}=249,4 \mathrm{~Hz}, \mathrm{C}_{\mathrm{Ar}}-4\right), 130,16\left(\mathrm{~d},{ }^{3} J_{C-F}=8,8 \mathrm{~Hz}, 2 \mathrm{xC}_{\mathrm{Ar}}-2,6\right)$, $128,00\left(\mathrm{C}_{\mathrm{Th}}\right), 123,70\left(\mathrm{~d},{ }^{4} J=2,8 \mathrm{~Hz}, \mathrm{C}_{\mathrm{Ar}}-1\right), 116,55\left(\mathrm{~d},{ }^{2} J_{C-F}=22,0 \mathrm{~Hz}, 2 \mathrm{xC}_{\mathrm{Ar}}-3,5\right), 114,48$ $\left(\mathrm{C}_{\mathrm{Th}}\right), 98,94\left(\mathrm{C}_{\mathrm{Th}}\right), 14,38\left(\mathrm{CH}_{3}\right), 14,40\left(\mathrm{CH}_{3}\right)$. Found, \%: C 58,16; $\mathrm{H} 4,20 ; \mathrm{N} 14,45$. $\mathrm{C}_{14} \mathrm{H}_{12} \mathrm{FN}_{3} \mathrm{OS}$. Calculated, C 58,12; H 4,18; N 14,52.

3-(3-(4-Fluorophenyl)-1,2,4-oxadiazol-5-yl)-4,5,6,7-tetrahydrobenzo[b]thiophen-2amine 3f. Yield $78 \%$. M.p. $=194-195{ }^{\circ} \mathrm{C} .{ }^{1} \mathrm{HNMR}\left(500 \mathrm{MHz}, \mathrm{DMSO}-d_{6}\right)$ ppm: $\delta 1,73$ (br. s, $4 \mathrm{H}, \mathrm{CH}_{2}$ ), 2,46 (br. s, 2H, $\mathrm{CH}_{2}$ ), 2,72 (br. s, $2 \mathrm{H}, \mathrm{CH}_{2}$ ), 7,35 (t, $J=8,6 \mathrm{~Hz}, 2 \mathrm{H}, \mathrm{H}_{\mathrm{Ar}^{-}}$ 3,5), 7,67 (br. s, $2 \mathrm{H}, \mathrm{NH}_{2}$ ), 8,15 (dd, J = 8,6, 5,4 Hz, 2H, H $\left.\mathrm{Ar}_{\mathrm{Ar}}-2,6\right) .{ }^{13} \mathrm{C}$ NMR $(126 \mathrm{MHz}$, DMSO-d $\left.d_{6}\right) \mathrm{ppm}: \delta 173,33\left(\mathrm{C}_{\text {Oxadz }}-5\right), 165,80\left(\mathrm{C}_{\text {Oxadz }}-3\right), 163,03\left(\mathrm{C}_{\mathrm{Th}}\right), 160,97\left(\mathrm{~d},{ }^{1} J_{C-F}=\right.$ $\left.249,4 \mathrm{~Hz}, \mathrm{C}_{\mathrm{Ar}}-4\right), 130,37\left(\mathrm{C}_{\mathrm{Th}}\right), 130.15\left(\mathrm{~d},{ }^{3} J_{C-F}=8,9 \mathrm{~Hz}, 2 \mathrm{xC}_{\mathrm{Ar}}-2,6\right), 123.70\left(\mathrm{~d},{ }^{4} \mathrm{~J}=2,8\right.$ $\left.\mathrm{Hz}, \mathrm{C}_{\mathrm{Ar}}-1\right), 117,85\left(\mathrm{C}_{\mathrm{Th}}\right), 116.56\left(\mathrm{~d},{ }^{2} J_{C-F}=22,0 \mathrm{~Hz}, 2 \mathrm{xC}_{\mathrm{Ar}}-3,5\right), 97,60\left(\mathrm{C}_{\mathrm{Th}}\right), 26,15\left(\mathrm{CH}_{2}\right)$, $24,28\left(\mathrm{CH}_{2}\right), 23,19\left(\mathrm{CH}_{2}\right), 22,66\left(\mathrm{CH}_{2}\right)$. Found, \%:C 60,99; $\mathrm{H} 4,46 ; \mathrm{N} 13,29 . \mathrm{C}_{16} \mathrm{H}_{14} \mathrm{FN}_{3} \mathrm{OS}$. Calculated, C 60,94; H 4,47; N 13,32.

3-(3-(4-Chlorophenyl)-1,2,4-oxadiazol-5-yl)-4,5-dimethylthiophen-2-amine 3g. Yield $74 \%$. M.p. $=216-217{ }^{\circ} \mathrm{C} .{ }^{1} \mathrm{HNMR}\left(400 \mathrm{MHz}, \mathrm{DMSO}-d_{6}\right) \mathrm{ppm:} \delta 2,14\left(\mathrm{~s}, 3 \mathrm{H}, \mathrm{CH}_{3}\right)$, 2,24 (s,3H, $\mathrm{CH}_{3}$ ), 7,60 (d, 2H, $\left.J=8,3 \mathrm{~Hz}, \mathrm{H}_{\mathrm{Ar}}-3,5\right), 7,67$ (br. s, $2 \mathrm{H}, \mathrm{NH}_{2}$ ), 8,13 (d, 2H, $J=$ $\left.8,3, \mathrm{H}_{\mathrm{Ar}}-2,6\right) .{ }^{13} \mathrm{C}$ NMR (101 MHz, DMSO- $\left.d_{6}\right) \mathrm{ppm}$ : $\delta$ 173,51 ( $\left.\mathrm{C}_{\text {Oxadz }}-5\right), 165,80\left(\mathrm{C}_{\text {Oxadz }}-3\right)$, $160,52\left(\mathrm{C}_{\mathrm{Th}}\right), 136,42\left(\mathrm{C}_{\mathrm{Th}}\right), 129,63\left(2 \mathrm{xC}_{\mathrm{Ar}}-3,5\right), 129,48\left(2 \mathrm{xC}_{\mathrm{Ar}}-2,6\right), 127,99\left(\mathrm{C}_{\mathrm{Ar}}-4\right), 125,99$ $\left(\mathrm{C}_{\mathrm{Ar}}-1\right), 114,53\left(\mathrm{C}_{\mathrm{Th}}\right), 99,88\left(\mathrm{C}_{\mathrm{Th}}\right), 14,40\left(\mathrm{CH}_{3}\right), 12,44\left(\mathrm{CH}_{3}\right)$. Found, \%: C 54,92; H 3,98; N 13,77. $\mathrm{C}_{14} \mathrm{H}_{12} \mathrm{ClN}_{3} \mathrm{OS}$. Calculated, C 54,99; H 3,96; N 13,74.

3-(3-(4-Chlorophenyl)-1,2,4-oxadiazol-5-yl)-4,5,6,7-tetrahydrobenzo[b]thiophen-2amine 3h. Yield $73 \%$. M.p. $=188-189{ }^{\circ} \mathrm{C} .{ }^{1} \mathrm{HNMR}\left(400 \mathrm{MHz}, \mathrm{DMSO}-d_{6}\right)$ ppm: $\delta 1,74$ (br. s, 
$4 \mathrm{H}, \mathrm{CH}_{2}$ ), 2,47 (br. s, $2 \mathrm{H}, \mathrm{CH}_{2}$ ), 2,72 (br. s, $2 \mathrm{H}, \mathrm{CH}_{2}$ ), 7,62-7,64 (m, 3H, $\mathrm{H}_{\mathrm{Ar}}-3,4,5$ ), 7,68 (br. s, $\left.2 \mathrm{H}, \mathrm{NH}_{2}\right), 8,12\left(\mathrm{~d}, J=8,6 \mathrm{~Hz}, 2 \mathrm{H}, \mathrm{H}_{\mathrm{Ar}}-2,6\right) .{ }^{13} \mathrm{C} \mathrm{NMR}\left(101 \mathrm{MHz}, \mathrm{DMSO}-d_{6}\right) \mathrm{ppm}: \delta$ 173,41 ( $\left.\mathrm{C}_{\text {Oxadz }}-5\right), 165,82\left(\mathrm{C}_{\text {Oxadz }}-3\right), 161,08\left(\mathrm{C}_{\mathrm{Th}}\right), 136,38\left(\mathrm{C}_{\mathrm{Th}}\right), 130,35\left(\mathrm{C}_{\mathrm{Ar}}-4\right), 129,58$ $\left(2 \mathrm{xC}_{\mathrm{Ar}}-3,5\right), 129,45\left(2 \mathrm{xC}_{\mathrm{Ar}}-2,6\right), 126,01\left(\mathrm{C}_{\mathrm{Ar}}-1\right), 117,90\left(\mathrm{C}_{\mathrm{Th}}\right), 97,55\left(\mathrm{C}_{\mathrm{Th}}\right), 26,15\left(\mathrm{CH}_{2}\right)$, $24,29\left(\mathrm{CH}_{2}\right), 23,18\left(\mathrm{CH}_{2}\right), 22,65\left(\mathrm{CH}_{2}\right)$. Found, \%: C 57,98; $\mathrm{H} 4,28 ; \mathrm{N} 12,57$. $\mathrm{C}_{16} \mathrm{H}_{14} \mathrm{ClN}_{3} \mathrm{OS}$. Calculated, C 57,92; $\mathrm{H} 4,25 ; \mathrm{N} 12,66$.

3-(3-(2-Chlorophenyl)-1,2,4-oxadiazol-5-yl)-4,5-dimethylthiophen-2-amine $3 \mathrm{i}$. Yield $69 \%$. M.p. $=166-167{ }^{\circ} \mathrm{C} .{ }^{1} \mathrm{HNMR}(400 \mathrm{MHz}$, DMSO-d $)$ ppm: $\delta 2,14\left(\mathrm{~s}, 3 \mathrm{H}, \mathrm{CH}_{3}\right)$, $2.26\left(\mathrm{~s}, 3 \mathrm{H}, \mathrm{CH}_{3}\right), 7,50-7,61\left(\mathrm{~m}, 5 \mathrm{H}, \mathrm{H}_{\mathrm{Ar}}-3,4,5+\mathrm{NH}_{2}\right), 8,03\left(\mathrm{~m}, 1 \mathrm{H}, \mathrm{H}_{\mathrm{Ar}}-6\right) .{ }^{13} \mathrm{C} \mathrm{NMR}(101$ MHz, DMSO- $\left.d_{6}\right)$ ppm: $\delta$ 172,84 ( $\left.\mathrm{C}_{\text {Oxadz }}-5\right), 165,47\left(\mathrm{C}_{\text {Oxadz }}-3\right), 160,42\left(\mathrm{C}_{\mathrm{Th}}\right), 132,67\left(\mathrm{C}_{\mathrm{Th}}\right)$, $132,31\left(\mathrm{C}_{\mathrm{Ar}}\right), 131,23\left(2 \mathrm{xC}_{\mathrm{Ar}}\right), 128,00\left(2 \mathrm{xC}_{\mathrm{Ar}}\right), 126,36\left(\mathrm{C}_{\mathrm{Ar}}\right), 114,67\left(\mathrm{C}_{\mathrm{Th}}\right), 98,91\left(\mathrm{C}_{\mathrm{Th}}\right)$, $14,38\left(\mathrm{CH}_{3}\right), 12,43\left(\mathrm{CH}_{3}\right)$. Found, \%: C 54,91; $\mathrm{H} 3,99 ; \mathrm{N} 13,76 . \mathrm{C}_{14} \mathrm{H}_{12} \mathrm{ClN}_{3} \mathrm{OS}$. Calculated, C 54,99; H 3,96; N 13,74.

3-(3-(2-Chlorophenyl)-1,2,4-oxadiazol-5-yl)-4,5,6,7-tetrahydrobenzo[b]thiophen-2-

amine 3j. Yield $71 \%$. M.p. $=183-184{ }^{\circ} \mathrm{C} .{ }^{1} \mathrm{HNMR}\left(400 \mathrm{MHz}, \mathrm{DMSO}-d_{6}\right)$ ppm: $\delta 1,73$ (br. s, $4 \mathrm{H}, \mathrm{CH}_{2}$ ), 2,47 (br. s, $2 \mathrm{H}, \mathrm{CH}_{2}$ ), 2,74 (br. s, $2 \mathrm{H}, \mathrm{CH}_{2}$ ), 7,48-7,56 (m, 3H, $\mathrm{H}_{\mathrm{Ar}}-3,4,5$ ), 7,63 (br. s, $\left.2 \mathrm{H}, \mathrm{NH}_{2}\right), 8,03$ (d, $\left.J=7,6 \mathrm{~Hz}, 2 \mathrm{H}, \mathrm{H}_{\mathrm{Ar}}-2,6\right) \cdot{ }^{13} \mathrm{C}$ NMR (101 MHz, DMSO-d 6 ) ppm: $\delta 172,76\left(\mathrm{C}_{\text {Oxadz }}-5\right), 165,52\left(\mathrm{C}_{\text {Oxadz }}-3\right), 160,09\left(\mathrm{C}_{\mathrm{Th}}\right), 132,63\left(2 \times \mathrm{C}_{\mathrm{Ar}}\right), 132,29\left(\mathrm{C}_{\mathrm{Ar}}\right)$, $131,20\left(\mathrm{C}_{\mathrm{Ar}}\right), 130,36\left(\mathrm{C}_{\mathrm{Th}}\right), 127,97\left(\mathrm{C}_{\mathrm{Ar}}\right), 126,39\left(\mathrm{C}_{\mathrm{Ar}}\right), 118,05\left(\mathrm{C}_{\mathrm{Th}}\right), 97,59\left(\mathrm{C}_{\mathrm{Th}}\right), 26,15$ $\left(\mathrm{CH}_{2}\right), 24,29\left(\mathrm{CH}_{2}\right), 23,21\left(\mathrm{CH}_{2}\right), 22,67\left(\mathrm{CH}_{2}\right)$. Found, \%: C 57,82; $\mathrm{H} \mathrm{4,31;} \mathrm{N} \mathrm{12,71.}$ $\mathrm{C}_{16} \mathrm{H}_{14} \mathrm{ClN}_{3} \mathrm{OS}$. Calculated, C 57,92; H 4,25; N 12,66.

This work was supported by the National Research Foundation of Ukraine (Project No. 2020.01/0166) and the Ministry of Education and Science of Ukraine (Grant No. 0121U107777).

1. Huang Y., Dömling A. The Gewald multicomponent reaction // Mol Divers. 2011. Vol. 15. P. 3-33. DOI: https://doi.org/10.1007/s11030-010-9229-6

2. Wang K., Kim D., Dömling A. Cyanoacetamide MCR (III): Three-Component Gewald Reactions Revisited // J. Comb. Chem. 2010. Vol. 12, No. 1. P. 111-118. DOI: https://doi.org/10.1021/cc9001586

3. Bozorov K., Nie L. F., Zhao J. et al. 2-Aminothiophene scaffolds: Diverse biological and pharmacological attributes in medicinal chemistry // Eur. J. Med. Chem. 2017. Vol. 140. P. 465-493. DOI: https://doi.org/10.1016/j.ejmech.2017.09.039

4. Forero J. S. B., Jones J., Silva F. M. The Synthetic Potential and Chemical Aspects of the Gewald Reaction: Application in the Preparation of 2-Aminothiophenes and Related Heterocycles // Curr. Org. Synth. 2013. Vol. 10, No. 3. P. 347-365. DOI: https://doi.org/10.2174/1570179411310030002

5. Turkoglu G., Cinar M. E., Ozturk T. Thiophene-Based Organic Semiconductors // Top. Curr. Chem. (Z). 2017. Vol. 375, No. 6. Art. No. 84.

DOI: https://doi.org/10.1007/s41061-017-0174-z

6. Kim C., Choi K.S., Oh J.H. et al. The effects of octylthiophene ratio on the performance of thiophene based polymer light-emitting diodes // Sci. Adv. Mater. 2015. Vol. 7, P. 2401-2409. DOI: https://doi.org/10.1166/sam.2015.2669

7. Shah R., Verma P. K. Therapeutic importance of synthetic thiophene // Chem. Centr. J. 2018. Vol. 12, No. 1. Art. No. 137. DOI: https://doi.org/10.1186/s13065-018-0511-5 
8. Vitaku E., Smith D. T., Njardarson J.T. Analysis of the structural diversity, substitution patterns, and frequency of nitrogen heterocycles among U.S. FDA approved pharmaceuticals // J. Med. Chem. 2014 Vol. 57, No 24. P. 10257-10274. DOI: https://doi.org/10.1021/jm501100b

9. Chawla G. 1,2,4-Oxadiazole as a Privileged Scaffold for Anti-inflammatory and Analgesic Activities: A Review // Mini Rev. Med. Chem. 2018. Vol. 18, No. 18. P. 1536-1547. DOI: https://doi.org/10.2174/1389557518666180524112050

10. Biernacki K., Daśko M., Ciupak O. et al. Novel 1,2,4-Oxadiazole Derivatives in Drug Discovery // Pharmaceuticals. 2020. Vol. 13, No. 6. P. 111. DOI: https://doi.org/10.3390/ph13060111

11. Pokhodylo N. T., Shyyka O. Ya., Matiychuk V.S. et al. New Convenient Strategy for Annulation of Pyrimidines to Thiophenes or Furans via the One-pot Multistep Cascade Reaction of 1H-Tetrazoles with Aliphatic Amines // ACS Comb. Sci. 2015. Vol. 17, No. 7. P. 399-403. DOI: https://doi.org/10.1021/co5001376

12. Shyyka O. Ya., Pokhodylo N. T., Slyvka Yu. I. et al. Understanding the tetrazole ring cleavage reaction with hydrazines: Structural determination and mechanistic insight // Tetrahedron Lett. 2018. Vol. 59, No. 12. P. 1112-1115. DOI: https://doi.org/10.1016/j.tetlet.2018.02.016

13. Pokhodylo N. T., Shyyka O. Ya., Savka R. D. et al. Novel Selected Tandem Transformations of the Amino and Carbonyl/Nitrile Groups in the Gewald Thiophenes // Phosphorus, Sulfur, and Silicon and the Related Elements. 2010. Vol. 185, No. 10. P. 2092-2100. DOI: https://doi.org/10.1080/10426500903496739

14. Pokhodylo N. T., Shyyka O. Ya., Obushak M. D. Ethyl 2-Aminothiophene-3-Carboxylates in the Synthesis of Isomeric Thienopyridines // Chem. Heterocycl. Compds. 2014. Vol. 50, No. 12. P. 1748-1755. DOI: https://doi.org/10.1007/s10593-015-1647-z

15. Pokhodylo N. T., Shyyka O. Ya., Obushak M. D. Facile and efficient one-pot procedure for thieno[2,3-e][1,2,3]triazolo[1,5-a]pyrimidines preparation // Synth. Commun. 2014. Vol. 44, No. 7. P. 1002-1006. DOI: https://doi.org/10.1080/00397911.2013.840729

16. Pokhodylo N. T., Shyyka O. Ya. 1-(5-(R-amino)-1,2,4-thiadiazol-3-yl)propan-2-ones convenient ketomethylenic reagents for the Gewald and Dimroth reactions // J. Het. Chem. 2014. Vol. 51, No. 5. P. 1487-1490. DOI: https://doi.org/10.1002/jhet.1719

17. Shyyka O., Pokhodylo N., Obushak M. Synthesis of novel substituted 3-(5-aryl-1,3,4oxadiazol-2-yl)thiophen-2-amines via Gewald reaction // Visnyk Lviv Univ. Ser. Chem. 2013. Iss. 54 (2). P. 225-229. DOI: http://old.chem.lnu.edu.ua/visnykk/2013/225-229.pdf

18. Shyyka O.Ya., Martyak R. L., Tupychak M.A. et al. Facile synthetic route to benzo $[c]$ chromenones and thieno[2,3-c]chromenones // Synth. Comm. 2017. Vol. 47, No. 24. P. 2399-2405. DOI: https://doi.org/10.1080/00397911.2017.1380833

19. Pokhodylo N. T., Shyyka O. Ya., Tupychak M. A. et al. Selectivity in domino-reaction of ortho-carbonyl azides with malononitrile dimer leading to [1,2,3]triazolo[1,5a]pyrimidines // Chem. Heterocycl. Compd. 2018. Vol. 54, No. 2. P. 209-212. DOI: https://doi.org/10.1007/s10593-018-2256-4

20. Shyyka O., Pokhodylo N., Finiuk N. et al. Anticancer activity evaluation of new thieno[2,3-d]pyrimidin-4(3H)-ones and thieno[3,2-d]pyrimidin-4(3H)-ones derivatives // Sci. Pharm. 2018. Vol 86, No. 3. E 28. DOI: https://doi.org/10.3390/scipharm86030028

21. Shyyka O., Pokhodylo N., Finiuk N. Anticancer activity evaluation of thieno[3,2$e][1,2,3]$ triazolo[1,5-a]pyrimidines and thieno[2,3-e][1,2,3]triazolo[1,5-a]pyrimidine derivatives // Biopolym. Cell. 2019. Vol. 35, No. 4. P. 321-330.

DOI: http://dx.doi.org/10.7124/bc.000A0F 
22. Shyyka O. Ya., Pokhodylo N. T., Palchykov V. A. et al. Cage-like amines in the green protocol of transannularthieno[2,3- $d]$ pyrimidinone formation as promising anticancer agents // Chem. Heterocycl. Compd. 2020. Vol. 56, No. 6. P. 793-799.

DOI: https://doi.org/10.1007/s10593-020-02732-2

23. Gewald K., Schinke E., Bottcher H. 2-Amino-thiophene aus methylenaktiven nitrilen, carbonylverbindungen und schwefel // Chem. Ber. 1966. Vol. 99, P. 94-100.

DOI: https://doi.org/10.1002/cber.19660990116

24. Tupychak M. A., Shyyka O. Ya., Pokhodylo N.T. et al. Nitrileimines as an alternative to azides in base-mediated click [3+2]cycloaddition with methylene active nitriles // RSC Adv. 2020. Vol. 10, No. 23. P. 13696-13699. DOI: https://doi.org/10.1039/D0RA01417F

\title{
СИНТЕЗ НОВИХ ПОХІДНИХ 3-(1,2,4-ОКСАДІАЗОЛ-5-ІЛ)-2- АМІНОТІОФЕНІВ РЕАКЦІСЮ ІЕВАЛЬДА
}

\section{О. Шийка, М. Свириденко, М. Тупичак, Н. Походило, М. Обушак}

\author{
Львівський національний університет імені Івана Франка, \\ вул. Кирила і Мефодія, 6, 79005 Львів, Україна \\ e-mail: shiyka.olya@gmail.com
}

Для синтезу нових заміщених 3-(1,2,4-оксадіазол-5-іл)-2-амінотіофенів використано однореакторну мультикомпонентну реакцію Гевальда. Цей синтетичний протокол передбачає взаємодію нових реактантів - (3-арил-[1,2,4]оксадіазол-5-іл)ацетонітрилів з метиленактивними карбонільними сполуками (циклогексанон, етилметилкетон) і сіркою за наявності морфоліну як каталізатора. Таким способом одержано нові, раніше не описані, функціоналізовані похідні 3(1,2,4-оксадіазол-5-іл)-2-амінотіофени 3 високими виходами та чистотою: 4,5-диметил-3-(3-феніл1,2,4-оксадіазол-5-іл) тіофен-2-амін, $\quad$ 3-(3-феніл-1,2,4-оксадіазол-5-іл)-4,5,6,7-тетрагідробензо[ $b]$ тіофен-2-амін, 4,5-диметил-3-(3-(o-толіл)-1,2,4-оксадіазол-5-іл)тіофен-2-амін, 3-(3-(o-толіл)1,2,4-оксадіазол-5-іл)-4,5,6,7-тетрагідробензо[b]тіофен-2-амін, 3-(3-(4-фторофеніл)-1,2,4-оксадіазол5-іл)-4,5-диметилтіофен-2-амін, 3-(3-(4-фторофеніл)-1,2,4-оксадіазол-5-іл)-4,5,6,7-тетрагідробензо[b]тіофен-2-амін, 3-(3-(4-хлорофеніл)-1,2,4-оксадіазол-5-іл)-4,5-диметилтіофен-2-амін, 3-(3-(4хлорофеніл)-1,2,4-оксадіазол-5-іл)-4,5,6,7-тетрагідробензо[b]тіофен-2-амін, $\quad$ 3-(3-(2-хлорофеніл)1,2,4-оксадіазол-5-іл)-4,5-диметилтіофен-2-амін, 3-(3-(2-хлорофеніл)-1,2,4-оксадіазол-5-іл)-4,5,6,7тетрагідробензо $[b]$ тіофен-2-амін. Показано можливість успішного варіювання замісників у ацетонітрилах, що $€$ перевагою для створення комбінаторних бібліотек амінотіофенового скафолду 3 1,2,4-оксадіазольним фрагментом 3 метою пошуку лікарських препаратів. Будову цільових амінотіофенів підтверджено методами ${ }^{1} \mathrm{H}$ та ${ }^{13} \mathrm{C}$ ЯМР-спектроскопії.

Одержані сполуки є перспективними для вивчення біологічної активності. Вони пройшли відбір для дослідження протиракової активності у рамках співпраці з Національним інститутом раку (США), а антимікробної активності - зі Спільнотою 3 відкриття протимікробних препаратів (CO-ADD) (Велика Британія, Австралія). реакції.

Ключові слова: 1,2,4-оксадіазол, 2-амінотіофени, реакція Гевальда, мультикомпонентні 